\title{
Multi-type hyper-spectral microscopic imaging system
}

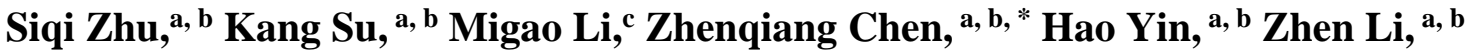 \\ ${ }^{a}$ Guangdong Provincial Key Laboratory of Optical Fiber Sensing and Communications, \\ Guangzhou, Guangdong 510632, China \\ ${ }^{b}$ Department of Optoelectronic Engineering, Jinan University, Guangzhou, Guangdong 510632 , \\ China \\ ${ }^{\mathrm{c} G u a n g z h o u L i s s ~ O p t i c a l ~ I n s t r u m e n t ~ C o ., ~ L t d, ~ G u a n g z h o u, ~ G u a n g d o n g, ~ 510095, ~ C h i n a ~}$
}

\begin{abstract}
We present a hyper-spectral microscopic imaging system capable of obtaining both transmission- and fluorescence-spectral images for biological measurements. For obtaining high quality images of samples with low self-fluorescence, large aperture optics and low vibration elements were implemented in the system. This study focused on obtaining different types of spectral information, i.e., transmission and fluorescence, to study the structure of the objects and observe the similarities and differences in composition at different positions. By employing this system to investigate bee pollen, we observed a special thin-film structure, which cannot be detected using conventional microscopic systems.
\end{abstract}

Keywords: LCTF; microscopy imaging; multi-type spectra; hyper-spectral imaging.

Corresponding author at: Institute of Optoelectronic Engineering, Jinan University, 601 Huangpu Avenue West, Guangzhou, China, 510632; Tel: +086 020-85224387; Fax: +086 02085224387; E-mail: tzqchen@jnu.edu.cn

\section{Introduction}

An imaging system is one of the most important tools required to study an object. However, conventional imaging systems only offer morphological information, which is an obvious disadvantage for scientific analysis. To overcome this limitation, spectral images, especially hyper-spectral images (HSI) that contain continuous and relatively complete records of spectral response to materials in a certain wavelength range, were developed. Objects with different chemical compositions have different spectral responses and can be distinguished from each other by their spectra. Accordingly, HSI systems are widely applied in various areas to identify the 
composition, status, or features of objects precisely. For example, Brigitte Mayinger et al. ${ }^{1}$ designed a system for the endoscopic detection of esophageal cancer in 2001 by using imaging spectroscopy, and Deborah Lau et al. ${ }^{2}$ reported a method for the elemental analysis of paintings using Raman spectral imaging. Other applications such as 3D image acquisition ${ }^{3}$ and automatic target detection using spectral images ${ }^{4}$ have also been reported. Moreover, the development of laser technology and 2D filter devices has enabled hyper-spectral imaging to be applied for microscopic analysis. ${ }^{5,6}$ For example, with a hyper-spectral microscopic imaging system, it is possible to obtain information about living cells ${ }^{7}$ or to distinguish normal and cancerous cells. ${ }^{8}$

There are several hyper-spectral technologies that can be used to achieve different spectral images, such as absorption spectroscopy, ${ }^{9}$ Fourier-transform infrared spectroscopy, ${ }^{10}$ and Raman spectroscopy. ${ }^{11}$ However, in general, some materials that are almost transparent or opaque are not suitable for absorption or transmission spectroscopy, and other materials do not generate sufficient self-fluorescence. Therefore, none of these technologies can be used to analyze all types of materials. Moreover, in some cases, the difference in the spectral response for two different materials is too subtle. Multi-type hyper-spectral detection can provide more comprehensive information, but most hyper-spectral imaging studies have focused only on a single type of spectral information. Only some researches about multi-mode hyper-spectral imaging system with fluorescence and reflectance detection were reported, ${ }^{12-15}$ and most of these are not the microscopic system. For improving the detection and obtaining more information from samples, we carry out the research of design and application of a multi-type hyper-spectral microscopic imaging system.

The simplest way to set up a multi-type hyper-spectral microscopic imaging system is to combine a fluorescence spectral system with a transmission spectral system because the structure 
and spectral detection range of the two systems are quite similar. In this study, we designed such a hyper-spectral microscopic imaging system, and used the bee pollen as test sample to investigate the detection ability of it due to its relative large size and strong self-fluorescence. Several studies on pollen have been reported, ${ }^{16-20}$ such as studies on obtaining morphologic information of bee pollen by using a scanning electron microscope (SEM). The SEM can obtain more morphologic details than the conventional microscope because of its high spatial resolution. For example, with a conventional bright-field microscope, the bee pollen of the jasmine flower has been observed as a pellet with a hole at the center, while morphologic information obtained using an SEM indicated that it is not a hole but rather a thin film in a fluted structure. ${ }^{21}$ However, SEMs are too expensive and can only obtain morphologic information. Though the spatial resolution of our proposed system is similar to that of a conventional bright-field microscope, we were able to determine that the center of the bee pollen is a thin film, rather than a hole, by using the proposed system alone. Based on the fluorescence spectral images, we could confirm that the composition of the thin film is the same as the rest of the pollen. Hence, we were able to obtain the transmission spectrum at the thin film in the bee pollen; it is difficult to obtain the transmission spectrum at other locations. These experimental results were based on multi-type spectral detection. Neither conventional microscopy nor a single-type hyper-spectral imaging system is capable of obtaining such data.

\section{Design of the multi-type hyper-spectral imaging system}

In our multi-type hyper-spectral microscopic system, the light passing through or being emitted from the sample will pass through the filter, focal lens, objects, and microscopic system to be imaged by the CCD. In this process, the light intensity will be decreased by certain factors. For studying light attenuation, a model was set up as follows: 
(i) The relationship between the light intensity on the sample $\left(I_{\text {sample }}\right)$ and the $\mathrm{CCD}\left(I_{C C D}\right)$ can be expressed as

$I_{C C D}=\frac{\left(1-\partial_{\lambda}\right)\left(1-\partial_{o}\right) I_{\text {sample }}}{\beta}$.

Here, $\beta$ is the magnification factor of the microscopic system. Only light with a particular wavelength can pass through the filter, while the other light will be absorbed or reflected; the loss coefficient of this process is represented as $\partial_{\lambda}$. The factor $\partial_{o}$ is used to describe the loss of the light escaping from the optical system.

(ii) The responsiveness $R$ of the CCD is related to the light intensity $I_{C C D}$, exposure time $t_{e}$, and the quality factor $K$ associated with the characteristics of the CCD. Thus, the factor $R$ can be described as

$R=K \times I_{C C D} \times t_{e}=\frac{K \times t_{e} \times\left(1-\partial_{\lambda}\right)\left(1-\partial_{o}\right) I_{\text {sample }}}{\beta}$

The higher the value of $R$ is, the higher the signal-to-noise ratio that can be obtained. As our microscopic system has a tunable filter, it has the significant disadvantage of a low optical transmission rate, especially when measuring samples with low self-fluorescence. Thus, in order to obtain high-quality images, the large-aperture elements, such as an objective with a large numerical aperture (low $\partial_{o}$ ) or a 2D filter with a wide field of view (low $\partial_{\lambda}$ ), can be used to improve the optical signal collection. The most commonly used 2D filters are the acousto-optic tunable filter (AOTF) and liquid-crystal tunable filter (LCTF), both of which have already been widely used in microscopic studies. ${ }^{22,23}$ In addition, an increase in the exposure time (long $t_{e}$ ) also has a beneficial impact on the intensity of the optical signal. However, if vibrations are present in the system, a longer exposure time leads to a loss in sharpness in the resulting images. Thus, 
mechanical vibrations should be avoided during spectral scanning. In this study, we chose an LCTF for our experimental system because of its high rejection rate of out-of-band transmission and the absence of mechanical vibrations.

The structure of the experimental setup is shown in Fig.1. It consisted of a hardware and software part.
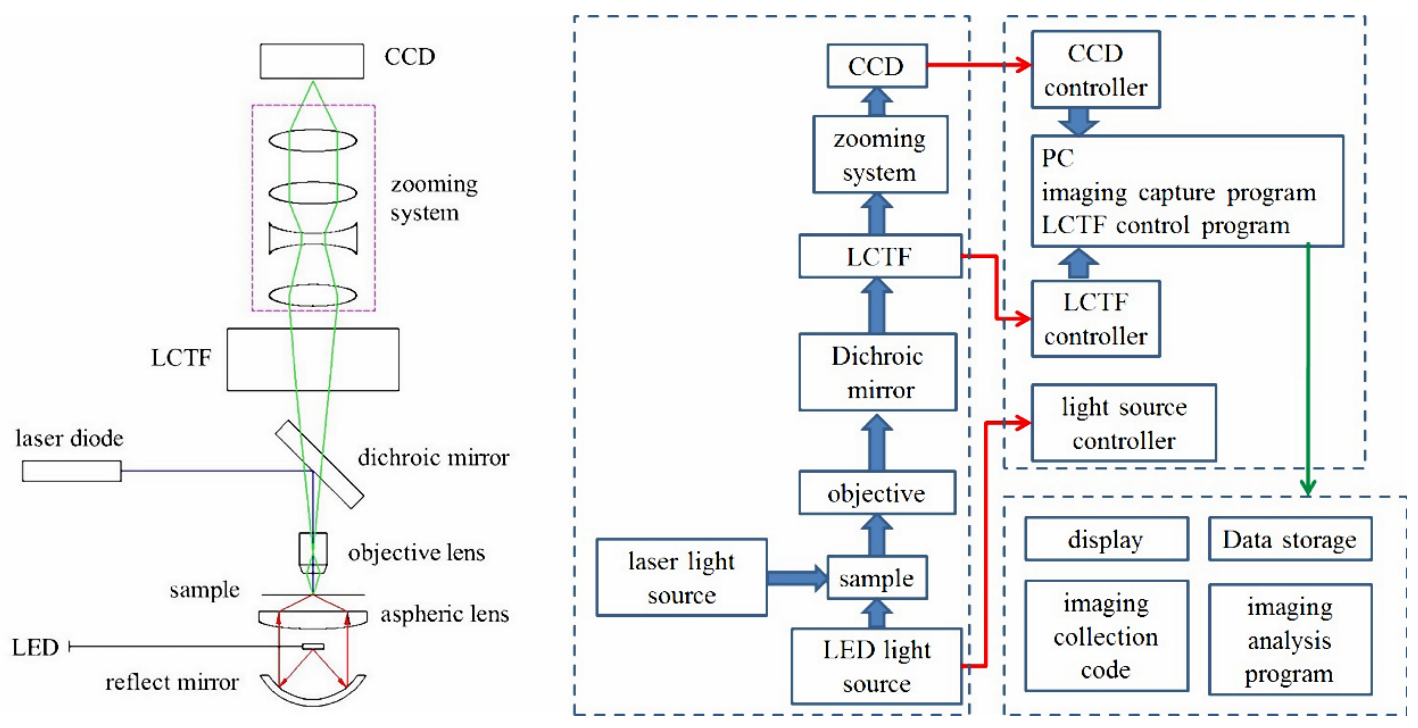

Fig. 1 Architecture of the hyper-spectral microscopic imaging system.

The hardware of the system comprises a 2/3-in CCD (SONY.INCEX view HAD ICX285) with an image size of $1360 \times 1024$ pixels, zooming lenses with magnification ranging from $1 \times$ to $7 \times$, an LCTF (CRI.INC, VariSpec VIS) covering the spectral range of 420-720 nm, a dichroic mirror (Thorlabs, Inc., DMLP425R) to separate the laser and optical signal, a 10× infinity-corrected imaging microscope objective (Olympus, Inc., RMS10X) with a large numerical aperture (N.A. = 0.25), and two different light sources for spectroscopy. A $20 \mathrm{~mW}$ laser diode (LD) emitting at $\lambda=$ $410 \mathrm{~nm}$ was used to excite the fluorescence, and a catadioptric white LED light source was used for transmission spectroscopy. The optical characteristics of the laser, LED, and dichroic mirror are shown in Fig. 2. 

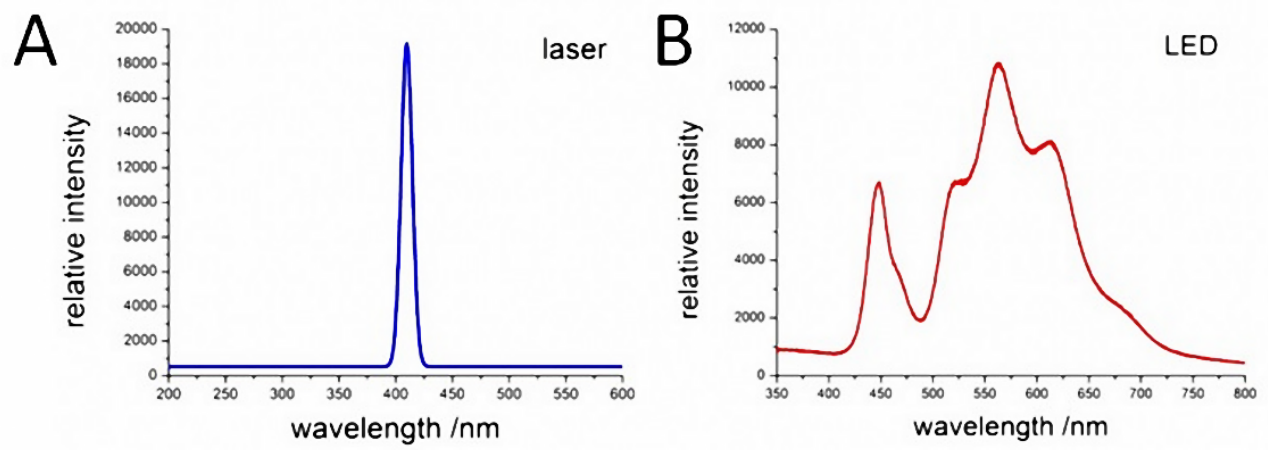

$45^{\circ} \mathrm{AOI}$ Unpolarized Light

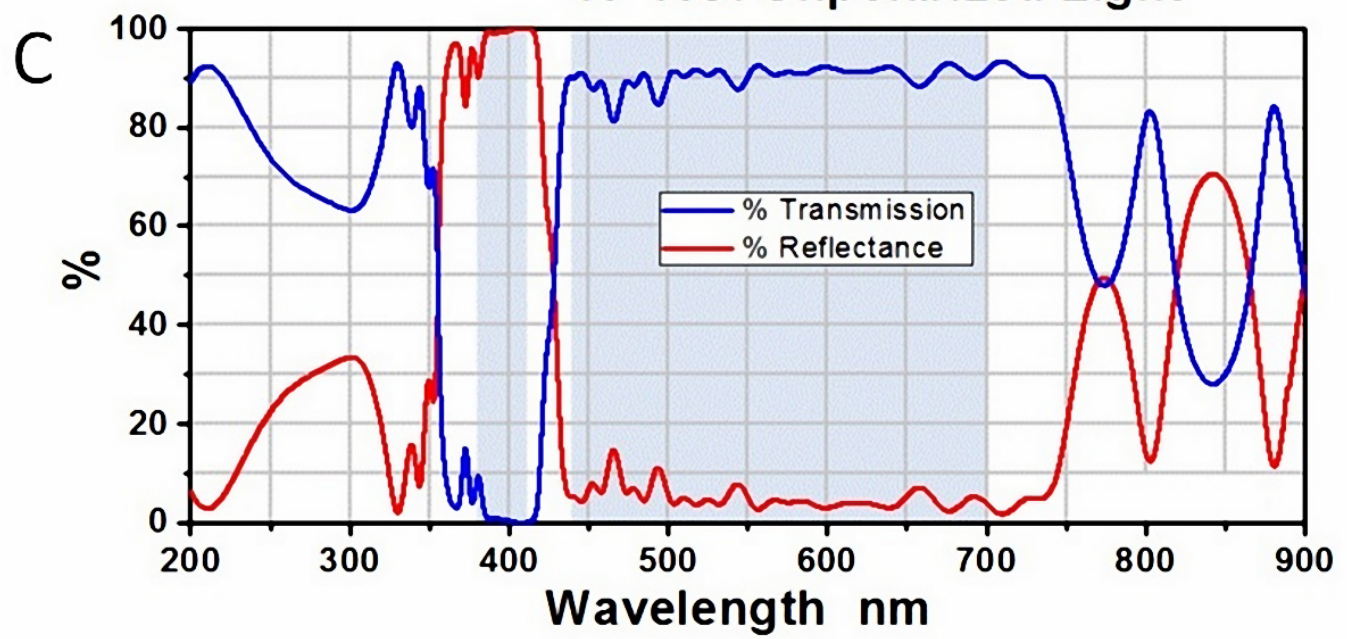

Fig. 2 Emission spectrum of the (A) laser and (B) LED light source. (C) The transmission and reflectance curves of the dichroic mirror with un-polarized light.

Software is used to control the gain factor, exposure time, sweep range, and step length. The minimum step length of spectral scanning and the maximum acquisition speed of the system are 5 $\mathrm{nm}$ and $50 \mathrm{~ms}$, respectively, as determined by the LCTF characteristics. The exposure time of the system is adjustable from 1/44000-115 s. The time series of the CCD and LCTF are shown in Fig. 3. We apply median filtering program to reduce the salt and pepper noise, and extract the spectral data by a MATLAB program in the computer. 


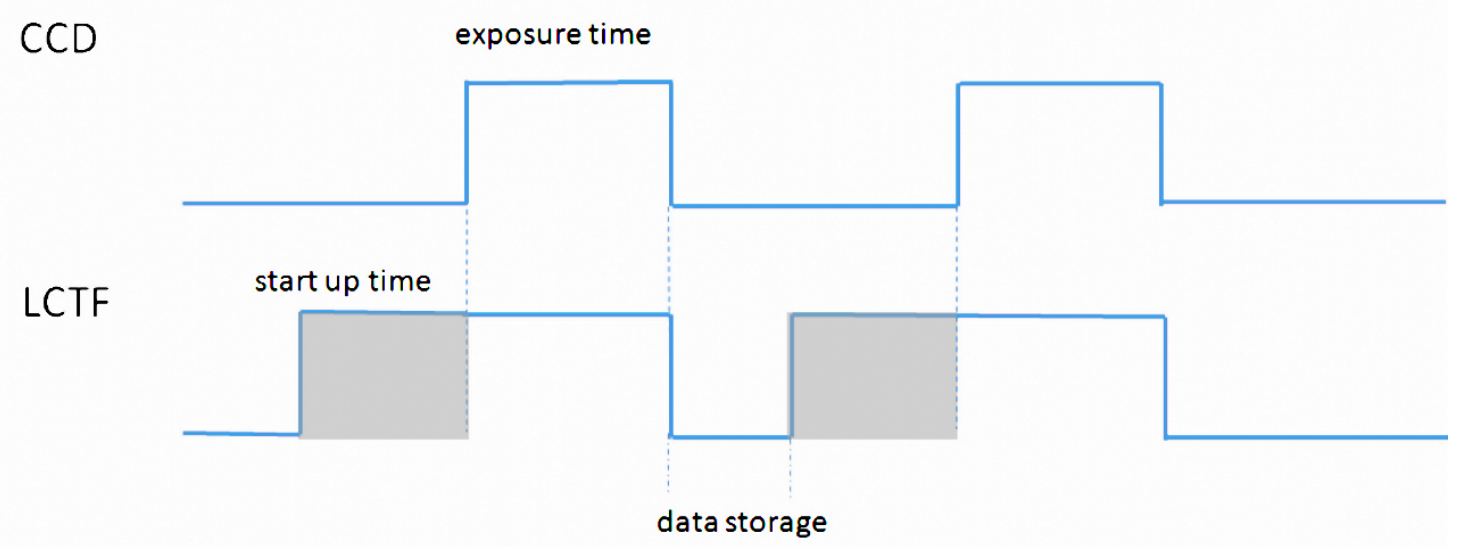

Fig. 3 Time series of CCD and LCTF.

When using the LED light source, the light is focused on the sample. A part of the light is scattered or absorbed by the sample, while another part enters the system, is filtered by the LCTF, and imaged by the CCD. The result is a bright-field image at a spectral range of $420 \mathrm{~nm}-720 \mathrm{~nm}$; we obtain the transmission rate by comparing the light intensities of the background and sample. On the other hand, when using the laser, the fluorescence is only emitted from the sample, and the background is dark. We obtain the fluorescence spectra from the variation of the emitted intensity with respect to the wavelength.

\section{Experimental results and analysis}

Samples of dried bee pollen of the jasmine flower were used to test the system. Samples collected from the Beijing Botanical Garden were offered by the Institute of Apicultural Research, CAAS (Chinese Academy of Agricultural Sciences). Bee pollen can be easily observed with a lowmagnification objective owing to its relatively large size. Furthermore, it contains numerous proteins, lipids and reducing sugar, which can generate high-efficiency self-fluorescence. ${ }^{24-28}$ All of these make it easy to observe with the hyper-spectral imaging system.

First, a bright-field image, illuminated by the LED, and a dark field image, excited by the laser, were obtained at a wavelength of $550 \mathrm{~nm}$ (see Fig. 4). 


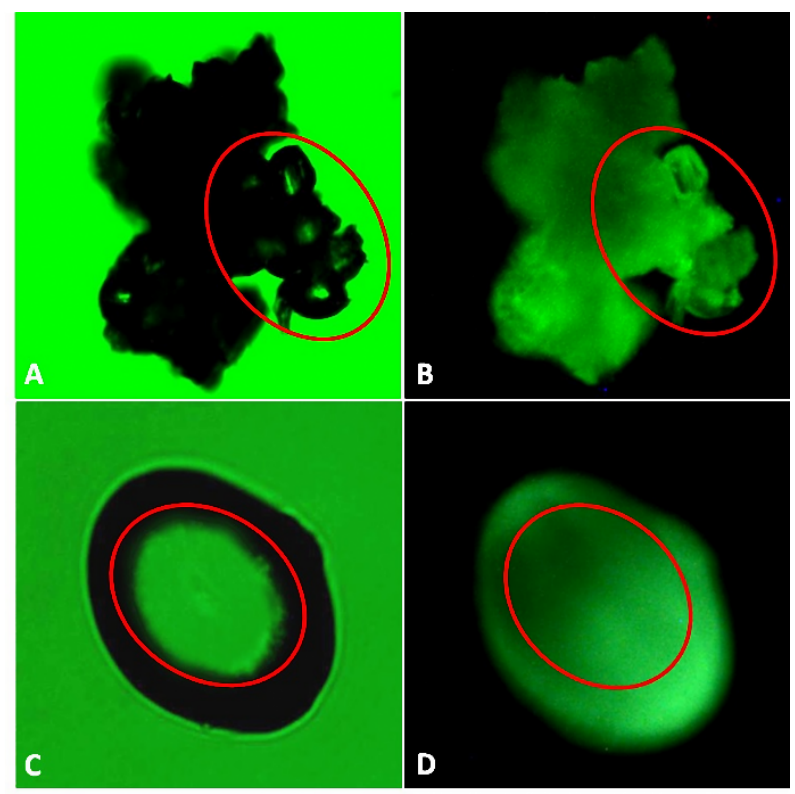

Fig. 4 (A) Bright-field transmission-spectral image of an agglomerate of bee pollen pellets, (B) dark-field fluorescence-spectral image of an agglomerate of bee pollen pellets, (C) bright-field transmission-spectral image of a single pellet of bee pollen and (D) dark-field fluorescence-spectral image of a single pellet of bee pollen. All images were taken at a wavelength of $550 \mathrm{~nm}$.

Both of transmission and fluorescence spectral images of an agglomerate and a single pellet of bee pollen were taken by the hyper-spectral microscopic imaging system. Bee pollen is imaged as a pellet with a hole at the center when it was illuminated by the LED light source (Fig. A, C). However, the hole does not appear in the fluorescence image (Fig. B, D). The spatial resolution of both imaging techniques should be the same because the same optical system is used, which implies that if a feature can be distinguished in image $\mathrm{A}$ and $\mathrm{C}$, it should also be distinguishable in image B and D.

To investigate this further, transmission-spectral images of a single pellet were obtained at different wavelengths (illuminated by the LED light source). In Fig. 5, we observe that the size of the hole changes with the wavelength. If the hole contains no material, the transmission rate of the entire hole should be the same at different wavelengths $(T=1)$, and its apparent size would not 
change with the wavelength. Moreover, if the hole contains some material with a homogeneous distribution, the apparent size of the hole would not change with the wavelength, because the transmission rates at every point in the hole are the same at different wavelengths. Thus, the hole should contain some material, and the distribution of the material should be inhomogeneous if the apparent size of the hole would change with the wavelength. In addition, the hole is nearly transparent in the bright field, which indicates that the materials are relatively thin. Therefore, we can conclude that an inhomogeneous thin film exists at the center of bee pollen. Its existence also explains why no hole is observed in the fluorescence images: the thin film also emits selffluorescence when excited by the laser.

According to the analysis, this phenomenon can be described as follows. When taking an image at a particular wavelength with a low transmission rate, the thicker part of the film should become opaque, while the light intensity through the thinner part should be nearly unchanged. Therefore, the size of the hole in the image would appear to be reduced. Conversely, when taking an image at a particular wavelength with a high transmission rate, most of the light can pass through the film, including the thicker part. Thus, the apparent size of the hole in the image would increase.
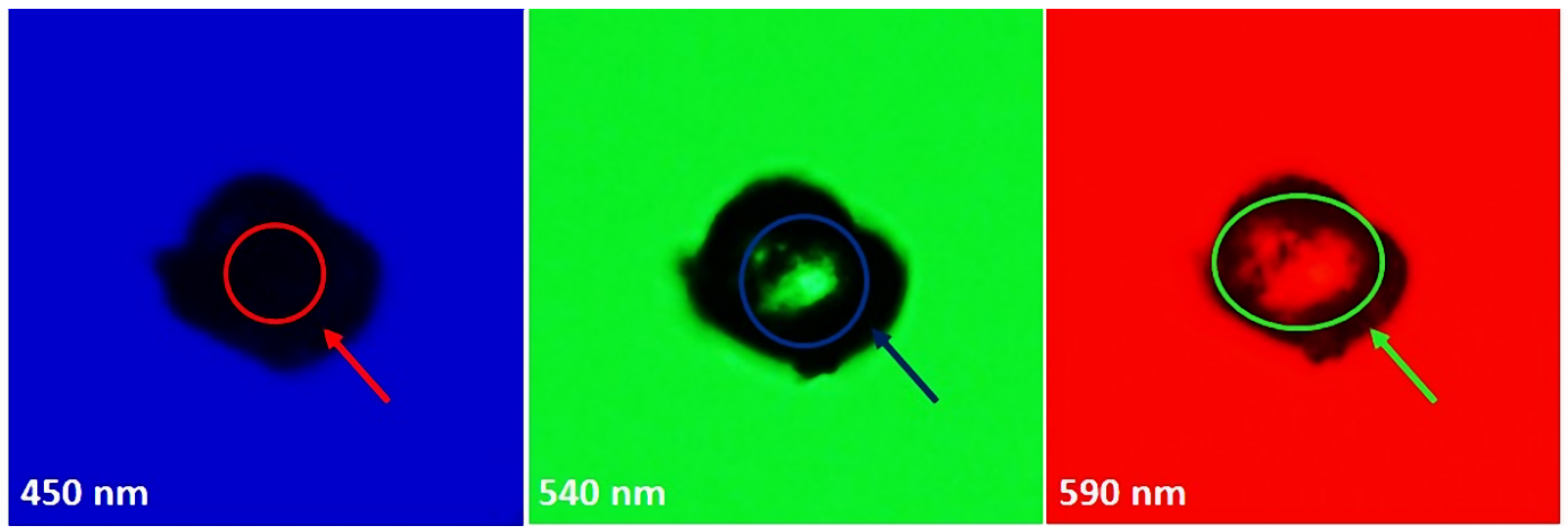

Fig. 5 Transmission-spectral images of a single pellet of bee pollen at different wavelengths.

We can ascertain more information from Fig. 5. 1) The tissues around the film are too thick to be detected with transmission. 2) The thickness of the film is inhomogeneous, and the edge of the 
film is thicker than the center of the film. 3) The transmission rate of the film at $590 \mathrm{~nm}$ is higher than that at $450 \mathrm{~nm}$.

To analyze the composition of the bee pollen, fluorescence images in a wavelength range of $420 \mathrm{~nm}-720 \mathrm{~nm}$ were obtained (excited by a laser at $410 \mathrm{~nm}$ ), as shown in Fig. 6 .

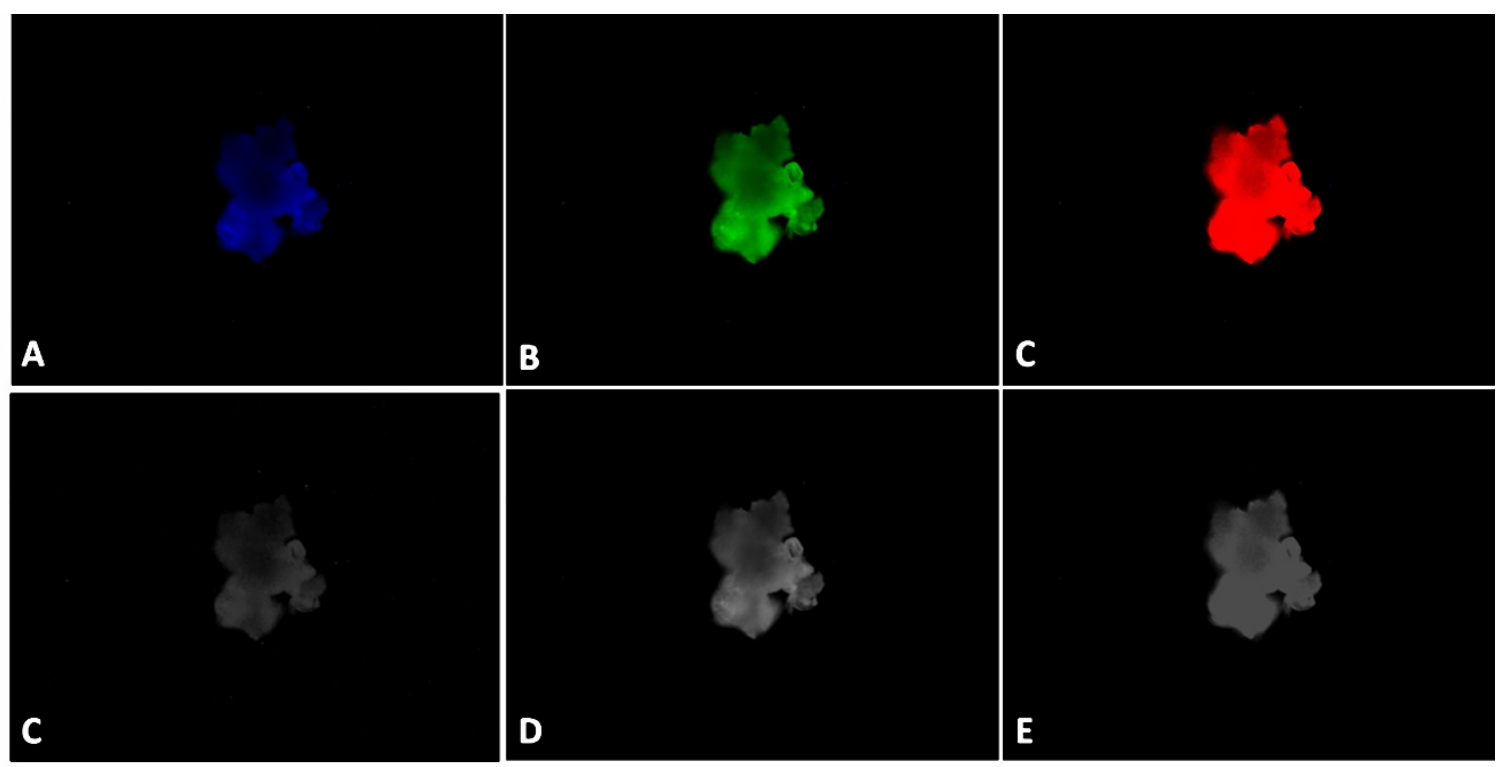

Fig. 6 Color-fluorescence and gray-fluorescence images of bee pollen $\left(\lambda_{\mathrm{a}}=\lambda_{\mathrm{c}}=480 \mathrm{~nm}, \lambda_{\mathrm{b}}=\lambda_{\mathrm{d}}=535 \mathrm{~nm}, \lambda_{\mathrm{c}}=\right.$ $\left.\lambda_{\mathrm{e}}=590 \mathrm{~nm}\right)$

Fluorescence spectra taken at different points of the pollen, including the thin film, are shown in Fig. 7. All spectra exhibit four peaks and are similar to each other.
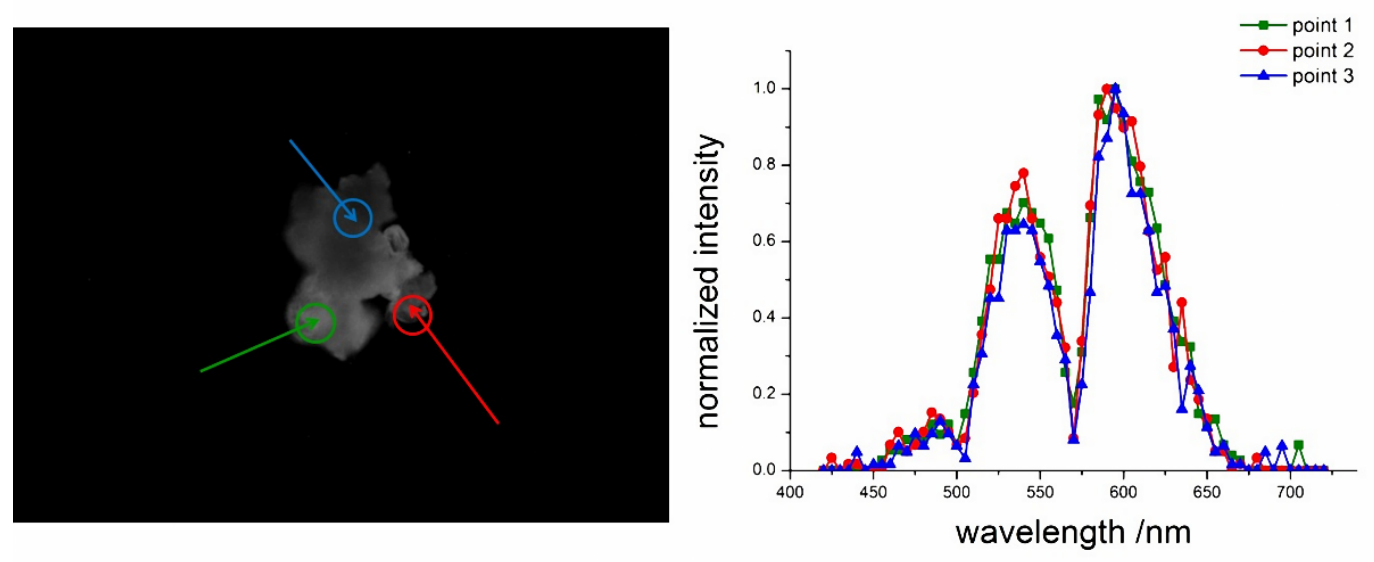

Fig. 7 Fluorescence spectra taken at different positions of bee pollen. 
The vertical axis of Figure 7 is the normalized intensity, which is expressed by the following formula:

$$
I_{\text {normalized }}=\frac{I-I_{\min }}{I_{\max }-I_{\min }}
$$

Here, $I$ is the intensity of the fluorescence at a certain wavelength, and $I_{\min }$ and $I_{\max }$ are the minimum and maximum intensities in the entire wavelength range, respectively. Because the gray values were proportional to the light intensity, they were used to replace light intensity in this experiment.

To investigate the detection precision, we recorded fluorescence spectra at sixty different positions on the pollen. The fluctuations of the position of each peak are shown in Fig. 8. The spectral resolution of this system is $5 \mathrm{~nm}$ because of the limitations of the LCTF; hence, the minimum fluctuation is $5 \mathrm{~nm}$.

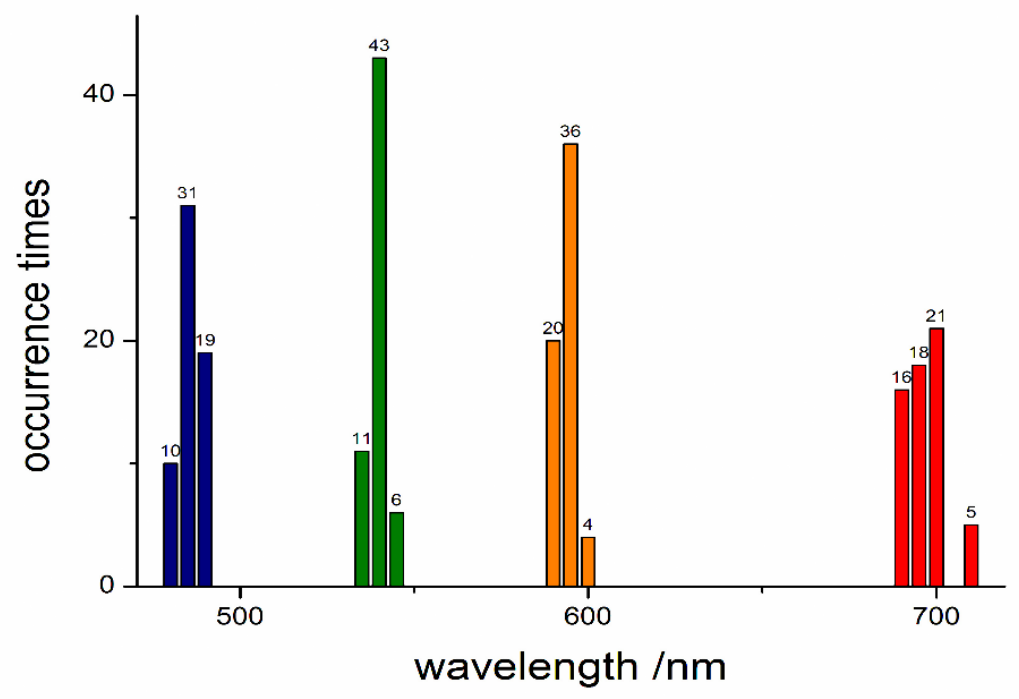

Fig. 8 Fluctuations of four fluorescence-peak positions.

The wavelengths of the peaks with the maximum occurrence rate were $485 \mathrm{~nm}, 540 \mathrm{~nm}, 595$ $\mathrm{nm}$, and $705 \mathrm{~nm}$, respectively. The maximum fluctuation was less than $\pm 10 \mathrm{~nm}$. Because of the 
large separation between the peaks, they could be distinguished from each other despite the shifts of $\pm 10 \mathrm{~nm}$.

The fluorescence spectra taken at multiple points indicated that the composition is the same throughout the bee pollen. Thus, the transmission spectra at the thin film and the rest of the pollen should also be the same. As the tissues around the film are too thick for transmission spectroscopy, we measured it at the position of the film where the thickness is low.

The transmission rate can be calculated using the following formula:

$$
T=\frac{I_{\text {sample }}}{I_{\text {background }}}
$$

Here, $T$ is the transmission rate, and $I_{\text {sample }}$ and $I_{\text {background }}$ are the light intensities from the sample and background, respectively. The transmission spectrum at the position of the film is shown in Fig. 9. The result was consistent with the analysis in Fig. 5.
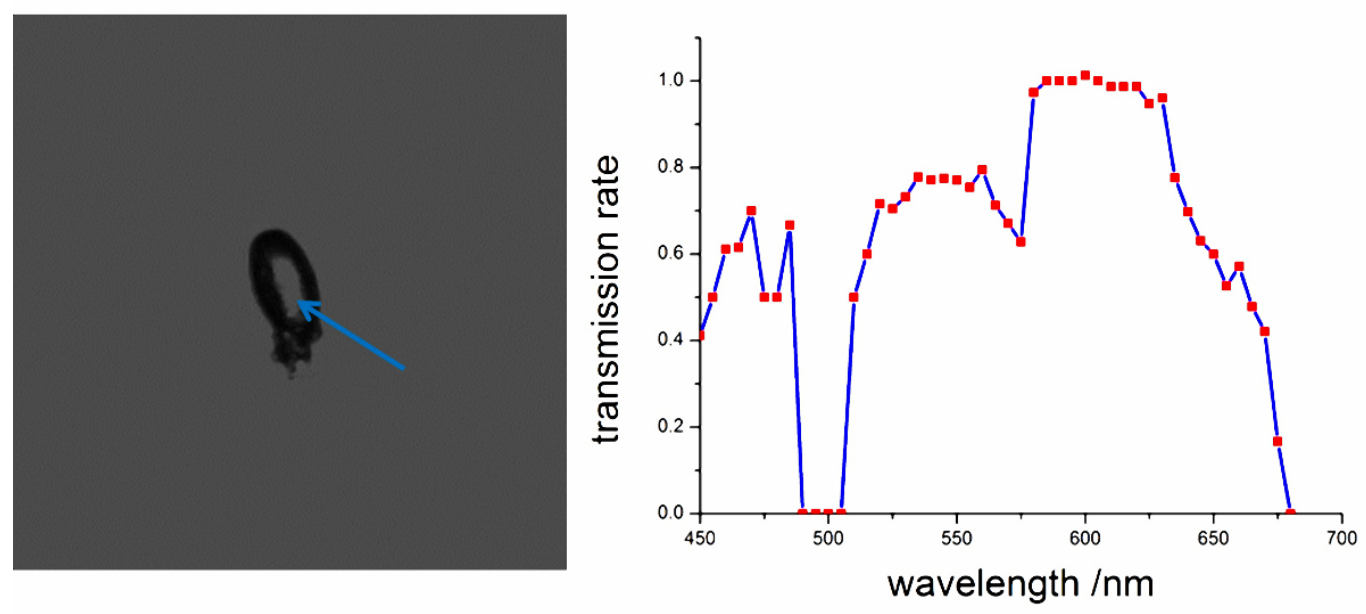

Fig. 9 Transmission spectrum of bee pollen at the thin film.

\section{Conclusion}

In this paper, we presented a hyper-spectral microscopic imaging system capable of obtaining both transmission- and fluorescence-spectral images, and studied bee pollen as a sample system with it. 
The experimental results showed that as a multi-type spectral detector, it can not only obtain more information but also take full advantage of the information for a comparative analysis. With this system, we obtained the transmission and fluorescence spectra of the sample, and found a special thin film in the bee pollen, while we cannot obtain so much information with a conventional microscopic system.

\section{Acknowledgments}

This work is partially supported by National Science Foundation (NSF) $(61475067,11404332$ );

Guangdong Project of Science and Technology Grants (2013B090600045, 2014B090903014, 2014B010131004, 2014B010124002, and 2015B090901014).

\section{References}

1. B. Mayinger, P. Horner, M. Jordan, C. Gerlach, T. Horbach, W. Hohenberger, E.G. Hahn, Lightinduced autofluorescence spectroscopy for the endoscopic detection of esophageal cancer, Gastrointest Endosc, 54 (2001) 195-201.

2. D. Lau, C. Villis, S. Furman, M. Livett, Multispectral and hyperspectral image analysis of elemental and micro-Raman maps of cross-sections from a 16th century painting, Anal Chim Acta, 610 (2008) $15-24$.

3. P. Latorre-Carmona, E. Sanchez-Ortiga, X. Xiao, F. Pla, M. Martinez-Corral, H. Navarro, G. Saavedra, B. Javidi, Multispectral integral imaging acquisition and processing using a monochrome camera and a liquid crystal tunable filter, Opt Express, 20 (2012) 25960-25969.

4. X.G. Sun, J.J. Cai, Z.Y. Xu, J.L. Zhang, Hyperspectral target detection based on improved automatic morphological endmember extraction method, Proc Spie, 8415 (2012). 
5. N. Mncwangi, I. Vermaak, A.M. Viljoen, Mid-infrared spectroscopy and short wave infrared hyperspectral imaging-A novel approach in the qualitative assessment of Harpagophytum procumbens and H. zeyheri (Devil's Claw), Phytochem Lett, 7 (2014) 143-149.

6. V. Studer, J. Bobin, M. Chahid, H.S. Mousavi, E. Candes, M. Dahan, Compressive fluorescence microscopy for biological and hyperspectral imaging, P Natl Acad Sci USA, 109 (2012) E1679-E1687.

7. Y. Hiraoka, T. Shimi, T. Haraguchi, Multispectral imaging fluorescence microscopy for living cells, Cell Struct Funct, 27 (2002) 367-374.

8. A.M. Siddiqi, H. Li, F. Faruque, W. Williams, K. Lai, M. Hughson, S. Bigler, J. Beach, W. Johnson, Use of hyperspectral imaging to distinguish normal, precancerous, and cancerous cells, Cancer Cytopathol, 114 (2008) 13-21.

9. L.J. Markstrom, G.A. Mabbott, Obtaining absorption spectra from single textile fibers using a liquid crystal tunable filter microspectrophotometer, Forensic Sci Int, 209 (2011) 108-112.

10. P. Lasch, W. Haensch, D. Naumann, M. Diem, Imaging of colorectal adenocarcinoma using FT-IR microspectroscopy and cluster analysis, Bba-Mol Basis Dis, 1688 (2004) 176-186.

11. C. Krafft, D. Codrich, G. Pelizzo, V. Sergo, Raman and FTIR microscopic imaging of colon tissue: a comparative study, J Biophotonics, 1 (2008) 154-169.

12. M.S. Kim, Y.R. Chen, P.M. Mehl, Hyperspectral reflectance and fluorescence imaging system for food quality and safety, T Asae, 44 (2001) 721-729.

13. M.S. Kim, B.K. Cho, C.C. Yang, K. Chao, A.M. Lefcourt, Y.R. Chen, Hyperspectral reflectance and fluorescence line-scan imaging system for online detection of fecal contamination on apples - art. no. 63810P, P Soc Photo-Opt Ins, 6381 (2006) P3810-P3810.

14. M.E. Martin, M. Wabuyele, M. Panjehpour, B. Overholt, R. DeNovo, S. Kennel, G. Cunningham, T. Vo-Dinh, An AOTF-based dual-modality hyperspectral imaging system (DMHSI) capable of simultaneous fluorescence and reflectance imaging, Med Eng Phys, 28 (2006) 149-155. 
15. S. Lenk, L. Chaerle, E.E. Pfundel, G. Langsdorf, D. Hagenbeek, H.K. Lichtenthaler, D. Van der Straeten, C. Buschmann, Multispectral fluorescence and reflectance imaging at the leaf level and its possible applications, J Exp Bot, 58 (2007) 807-814.

16. B. E. Vaissiere and S. B. Vinson, Pollen morphology and its effect on pollen collection by honey-bees, Apis-Mellifera L (Hymenoptera, Apidae), with special reference to upland cotton, GossypiumHirsutum L (Malvaceae), Grana, 33 (1994) 128-138.

17. K. N. Paudayal and I. Gautam, Scanning electron microscopic studies on surface pattern of the pollen loads from Apis cerana in Jajarkot District, Nepal Journal of Science and Technology, 12(2012) 340349.

18. V.G. Sagun, R.W.J.M. Van der Ham, Pollen morphology of the Flueggeinae (Euphorbiaceae, Phyllanthoideae), Grana, 42 (2003) 193-219.

19. M. Chica, Authentication of bee pollen grains in bright-field microscopy by combining one-class classification techniques and image processing, Microsc Res Techniq, 75 (2012) 1475-1485.

20. L. Pipino, M.C. Van Labeke, A. Mansuino, V. Scariot, A. Giovannini, L. Leus, Pollen morphology as fertility predictor in hybrid tea roses, Euphytica, 178 (2011) 203-214.

21. S. Wanke, J. Stockbridge, and G. Lamberton, “Jasmine pollen taken by SEM at 10000x,” 19

December, 2013 [http://www.izw-berlin.de/electron-microscopy.html].

22. E. S. Wachman, W. H. Niu, and D. L. Farkas, AOTF microscope for imaging with increased speed and spectral versatility, Biophys. J. 73 (1997) 1215-1222.

23. R. Lansford, G. Bearman, S.E. Fraser, Resolution of multiple green fluorescent protein color variants and dyes using two-photon microscopy and imaging spectroscopy, J Biomed Opt, 6 (2001) 311-318.

24. L.B. Almeida-Muradian, L.C. Pamplona, S. Coimbra, O.M. Barth, Chemical composition and botanical evaluation of dried bee pollen pellets, J Food Compos Anal, 18 (2005) 105-111.

25. M.G.R. Campos, S. Bogdanov, L.B. de Almeida-Muradian, T. Szczesna, Y. Mancebo, C. Frigerio, F. Ferreira, Pollen composition and standardisation of analytical methods, J Apicult Res, 47 (2008) 154161. 
26. T.H. Roulston, J.H. Cane, Pollen nutritional content and digestibility for animals, Plant Syst Evol, 222 (2000) 187-209.

27. L.B. Almeida-Muradian, L.C. Pamplona, S. Coimbra, O.M. Barth, Chemical composition and botanical evaluation of dried bee pollen pellets, J Food Compos Anal, 18 (2005) 105-111.

28. S. Ouchemoukh, H. Louaileche, P. Schweitzer, Physicochemical characteristics and pollen spectrum of some Algerian honeys, Food Control, 18 (2007) 52-58. 